\title{
Der Einfluss von druckinduzierten Trommelfellbewegungen auf vestibulospinale und vestibulookuläre Reaktionen bei Patienten mit Morbus Ménière
}

\author{
T. Petzold K.-B. Hüttenbrink Chr. Dörr \\ Klinik und Poliklinik für Hals-Nasen-Ohrenheilkunde, Universitätsklinikum Carl-Gustav Carus an der TU Dresden, \\ Deutschland
}

\section{Key Words}

Middle ear pressure · Ménière's disease · Posturography · Luzerner measuring platform

\section{Zusammenfassung}

Fragestellung: Mögliche Interaktionen zwischen Mittelohrdruck und Gleichgewichtsstörungen werden seit vielen Jahren diskutiert. Der Rolle der druckausgelösten Trommelfellbewegungen, die bei degenerativ fixierten Ossikelgelenken aufgrund des Hydraulikfaktors eine viel grössere Kraft ausüben als der direkte Druck an den Innenohrfenstern, wurde dabei nur geringe Aufmerksamkeit gewidmet. Patienten und Methodik: Wir untersuchten bei 19 Patienten mit einseitigem Morbus Ménière die durch wechselnde Drucke im äusseren Gehörgang (analog zur Tympanometrie) ausgelösten Interaktionen zwischen Trommelfellauslenkungen und vestibulären Reaktionen in der Elektronystagmographie und in der Posturographie. Ergebnisse und Schlussfolgerungen: Bei schnellen und langsamen Druckwechseln konnten zwischen -600 und +400 daPa weder auf dem gesunden noch auf dem erkrankten Ohr im Elektronystagmogramm Nystagmen nachgewiesen werden. Die posturographische Aufzeichnung der vestibulospinalen Reaktionen auf einer Luzerner Messplatte erwies sich dagegen als sensibler. Die Längenzunahme der Schwankungslinie zeigte bei beiden durchgeführten Druckwechseln auf dem kranken Ohr signifikante Unterschiede gegenüber der Messung bei Reizung des gesunden Ohres. Diese Methode ist somit der klassischen Prüfung des Hennebertschen Fistelsymptoms überlegen. Das vestibulospinale System reagiert möglicherweise empfindlicher als das vestibulookuläre auf Druckschwankungen der Perilymphe. Darüber hinaus könnte der nachgewiesene Zusammenhang der druckausgelösten Trommelfellbewegungen mit cochleovestibulären Symptomen bei einigen Patienten mit M. Ménière den Erfolg einer Paukenröhrcheneinlage erklären, da hiermit aussendruckinduzierte Trommelfellbewegungen und damit verbundene Perilymphschwankungen von vornherein unterbunden werden.

\section{The Influence of Pressure-Induced Ear Drum Movements on Vestibular Reactions in Patients Suffering from Ménière's Disease}

Objectives: Interactions between middle ear pressure and vestibular disturbances have been discussed for many years. Less attention has been paid to the influence of ear drum movements caused by pressure changes in the external auditory meatus. Because of the hydraulic amplification in case of fixed ossicular joints the impact of these movements is bigger than the direct pressure on the internal ear windows. Material and Patients: We examined the interaction between ear drum movements and vestibular reactions caused in the external auditory meatus of 19 patients suffering from unilateral Ménière's disease. Results and Conclusions: Both fast as well as slow pressure changes between -600 and +400 daPa were not able to induce nystagmus in the electronystagmogram of either the healthy or the damaged ear. Posturographic recordings of the vestibulospinal responses on the 'Luzerner measuring platform' were significantly more sensitive. The increase in length of the postural staggering line showed significant differences between the healthy and the damaged ear for fast and for slow pressure changes compared to calibration. In conclusion, the sensitivity of this examination is superior to the classical examination of Hennebert's fistula symptom. The vestibulospinal system is more sensitive towards pressure changes of the perilymph than the vestibuloocular system. The proven correlation between ear drum movements and internal ear symptomatology could explain the success of the treatment of patients suffering from Ménière's disease with tympanic grommets. Thus grommets prevent the pressure-induced ear drum movements and avoid perilymph movements induced by pressure changes.

Copyright $\odot 2001$ S. Karger AG, Basel

\begin{tabular}{ll}
\hline KARGER & ๑ 2001 S. Karger AG, Basel \\
Fax +4161306 12 34 & 1014-8221/00/0105-0207\$17.50/0 \\
$\begin{array}{l}\text { E-Mail karger@karger.ch } \\
\text { www.karger.com }\end{array}$ & $\begin{array}{l}\text { Accessible online at: } \\
\text { www.karger.com/journals/orn }\end{array}$
\end{tabular}

\footnotetext{
Dr. med. Torsten Petzold

Dr. med. Torsten Petzold
Klinik und Poliklinik für Hals-Nasen-Ohrenheilkunde

Universitätsklinikum Carl-Gustav Carus an der TU Dresden

Fetscherstrasse 74, D-01307 Dresden (Deutschland)

Tel. +49 3514584420 , Fax +49 3514584326
} 


\section{Influence des mouvements du tympan liés aux variations de pression sur les réactions vestibulo-spinales et vestibulo-oculaires de patients souffrant de la maladie de Ménière}

Chez les patients souffrant de la maladie de Ménière, les variations de pression entraînent des mouvements du tympan, influant ainsi sur les réactions vestibulaires. Objectifs: Si l'on s'est longtemps intéressé aux possibles interactions entre la pression de l'oreille moyenne et les troubles de l'équilibre, on a en revanche prêté moins d'attention à l'influence que peuvent exercer les mouvements du tympan dus aux variations de pression dans le conduit auditif externe. Or, lorsque les articulations ossiculaires sont bloquées, I'amplification hydraulique fait que ces mouvements ont un impact plus important que la pression directe exercée aux fenêtres de l'oreille interne. Méthodes et patients: Chez 19 malades souffrant d'un syndrome de Ménière unilatéral, nous avons étudié par ENG et posturographie la relation entre les réactions vestibulaires et les mouvements du tympan que déclenchent les variations de pression dans le conduit auditif externe. Résultats et conclusions: Qu'il s'agisse de variations de pression lentes ou rapides (entre -600 et $+400 \mathrm{daPa}$ ), de l'oreille saine ou déficiente, I'ENG n'a révélé aucun nystagmus. En revanche, les enregistrements posturographiques des réactions vestibulo-spinales sur la plate-forme de mesure de Lucerne ont été plus sensibles. L'allongement de la courbe de variation a montré des différences significatives entre l'oreille saine et déficiente, que ce soit lors de variations de pression rapides ou lentes. En conclusion, la précision de ce type d'examen se révèle nettement supérieure à celle de l'examen classique du signe de la fistule d'Hennebert (signe d'Hennebert). Le système vestibulo-spinal réagit apparemment de manière plus sensible que le système vestibulo-oculaire aux variations de pression du périlymphe. De plus, la relation que I'on a établie entre les mouvements du tympan dus aux variations de pression et les symptômes vestibulo-cochléaires pourrait expliquer les succès de la pose de diabolos chez certains patients souffrant de la maladie de Ménière. Ces diabolos empêchent les mouvements du tympan liés au changement de pression, évitant ainsi les mouvements du périlymphe.

\section{Einleitung}

Die Pathogenese des 1861 von P. Ménière erstmalig beschriebenen und nach ihm benannten Krankheitsbildes, charakterisiert durch die Trias aus attackenweise auftretendem Schwindel kombiniert mit Tinnitus und zunehmender, anfangs fluktuierender Schwerhörigkeit, konnte trotz umfangreicher wissenschaftlicher Forschung bis heute nicht eindeutig erklärt werden. Der endolymphatische Hydrops als Ursache, basierend auf einer Störung der quantitativen Beziehung zwischen Peri- und Endolymphvolumen durch Rückresorptionsstörungen der kaliumreichen Endolymphe und daraus folgender Erhöhung des osmotischen Drucks und Intoxikation der Sinneszellen durch die Vermischung beider Kompartimente, wird heute allgemein favorisiert. Dabei geht man aber von einer alleinigen Funktionsstörung des Innenohrs aus. Warum können aber nicht auch Veränderungen der kompliziert gestalteten Mittelohrstrukturen eine mögli- che Ursache der Erkrankung darstellen, diese unterhalten bzw. Innenohrsymptome verursachen?

Druckschwankungen im Mittelohr, auf die das Innenohr als hochempfindlicher Drucksensor äusserst sensibel reagiert, werden als Ursache cochleovestibulärer Symptome seit zirka 50 Jahren unter verschiedenen Gesichtspunkten diskutiert. Über eine Senkung des Umgebungsdruckes in einer Druckkammer und damit verbundenem Auspressen des Endolymphschlauches in den Saccus endolymphaticus durch den relativ erhöhten Mittelohrdruck konnten bei Morbus-Ménière-Patienten Besserungen des Hörvermögens [1] und Minderungen der Spontannystagmusaktivität $[2,3]$ beobachtet werden. Da das gleiche Phänomen auch bei ohrgesunden Probanden gefunden wurde, vermutete man auch eine direkte Übertragung auf die Perilymphe über das runde Fenster (Abb. 1).

Was allerdings bei dieser Betrachtungsweise ausser Acht gelassen wurde, ist der Tatbestand, dass über die Mittelohrstrukturen Trommelfell und Ossikelkette ein wesentlich stärkerer Antriebsmechanismus für Perilymphschwankungen potentiell vorhanden ist. Dieser wird beim Hören über das Flächenverhältnis von Trommelfell und Stapesfussplatte - den sogenannten Hydraulikfaktor - dafür genutzt, den am Trommelfell anliegenden Schalldruck zu verstärken und die unterschiedlichen Impedanzen von Luft und Perilymphe einander anzupassen. Beim Hören schwingen die Ossikel als funktionell fixierte, d.h. ungelenke Einheit um einen imaginären Drehpunkt, dessen Position sich frequenzabhängig ändert, kolbenförmig aus und ein. Die Vibrationen des Trommelfells und der Stapesfussplatte liegen dabei im nahezu unvorstellbaren Pico- und Nanometerbereich.

Andererseits wiederum reagiert das Ohr nicht nur auf Schalldrucke, sondern auch auf Änderungen des atmosphärischen Umgebungsdruckes, wie sie im täglichen Leben beim Fliegen und Liftfahren, Schneuzen und Pressen, aber auch bei den HNO-ärztlichen Tubenfunktionsprüfungen beobachtet werden. Das Trommelfell kann hierbei bis zu $1 \mathrm{~mm}$ bewegt werden, die zu messenden Druckänderungen betragen z.T. mehrere $100 \mathrm{~mm} \mathrm{H}_{2} \mathrm{O}$.

Diese im Vergleich zu den akustischen Vibrationen deutlich grösseren Verlagerungen werden aber in einer gesunden Ossikelkette durch das Gleiten der Gelenke abgefangen; das Innenohr wird von den Druckschwankungen der Aussenwelt abgekoppelt. Ist jedoch diese Gleitfunktion der Ossikelgelenke durch degenerative Veränderungen behindert, werden diese atmosphärischen Druckschwankungen ungehindert auf das Innenohr übertragen.

Aufgrund des Hydraulikfaktors entstehen bei nicht gleitfähigen Gelenken unphysiologisch grosse Stapesbewegungen und dadurch bedingt Perilymphverschiebungen [4-6]. Es ist vorstellbar, dass diese wiederum zur 


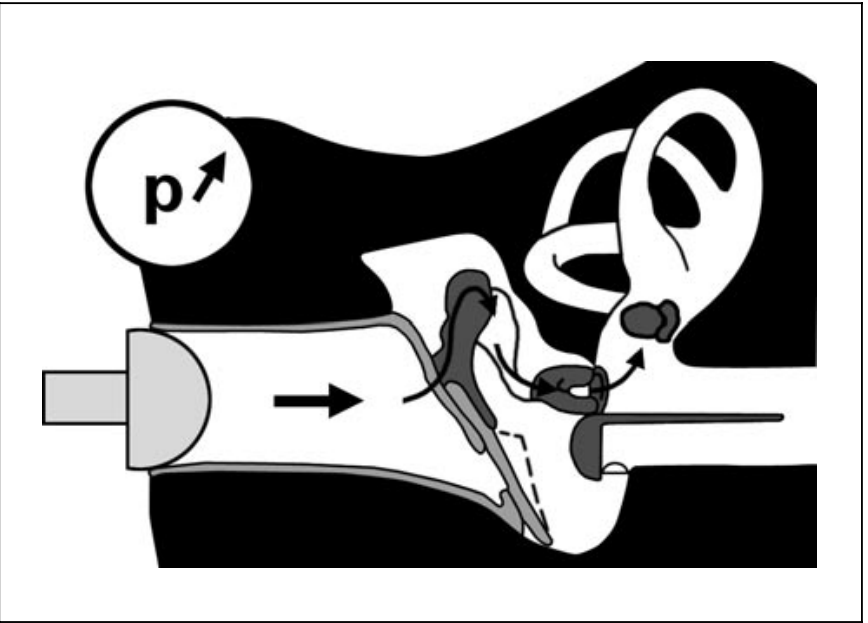

Abb. 1. Mögliche Übertragungswege atmosphärischer Druckschwankungen auf das Innenohr.

deutlichen Auslenkung der Cupula der einzelnen Bogengänge führen können.

Aus histologischen Untersuchungen ist bekannt, dass mit zunehmendem Alter degenerative Veränderungen der Ossikelkette zunehmen [7, 8]. Könnten nicht in diesen Fällen durch die permanent auf das $\mathrm{Ohr}$ einwirkenden und ungehindert ins Innenohr fortgeleiteten Druckschwankungen der Umgebung die empfindlichen Reiztransformatoren des Innenohres zunächst akut und später chronisch gereizt werden, was irgendwann zu deren Überlastung führt und die Innenohrsymptome Schwindel, Tinnitus und Hörstörung hervorruft?

Wir wollten daher bei Patienten mit einseitigem symptomatischem M. Ménière prüfen, ob eine Druckapplikation im Gehörgang zu einer Irritation der bei diesen Patienten möglicherweise besonders sensibilisierten Gleichgewichtsorgane führt.

\section{Methodik}

Um den möglichen Zusammenhang zwischen erheblichen, aber noch physiologischen Änderungen des an der Trommelfellebene anliegenden Luftdrucks und Irritationen der Innenohrfunktion näher zu untersuchen, führten wir bei 19 Patienten mit einem einseitigen M. Ménière und einer durchschnittlichen Erkrankungsdauer von 10,8 Jahren im beschwerdefreien Intervall Untersuchungen durch. Die Anfälle traten in Abständen von 2 Tagen bis zu 6 Monaten auf und dauerten von wenigen Minuten bis $\mathrm{zu} 36 \mathrm{~h}$ an. Nahezu alle Patienten gaben an, im Anfall unter heftigen Drehschwindelattakken, oft von vegetativen Symptomen begleitet, zu leiden. Bei der Erhebung einer ausführlichen Anamnese zur Dauer der Erkrankung, der Häufigkeit, Dauer und dem möglichen Auslösemechanismus der Anfälle sowie zu Tinnitus, Hörstörung und Druckgefühl im betroffenen Ohr wurde besonderer Wert auf das subjektive Empfinden von Schwindel bei Druckänderungen (Naseputzen, Gebirgsaufenthalte, Liftfahren, Flugreisen usw.) gelegt.

Der Einfluss statischen Mittelohrdrucks beim Morbus Ménière

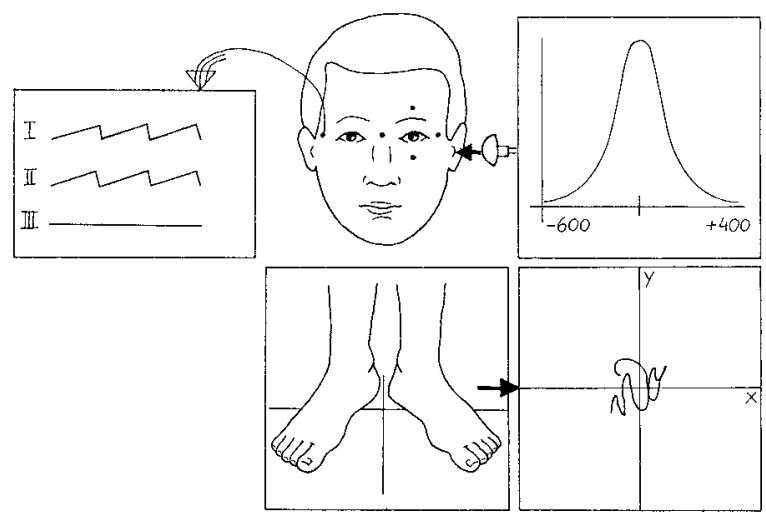

Abb. 2. Versuchsaufbau mit 3-Kanal-ENG, Vierfeldermessplatte und Tympanometer.

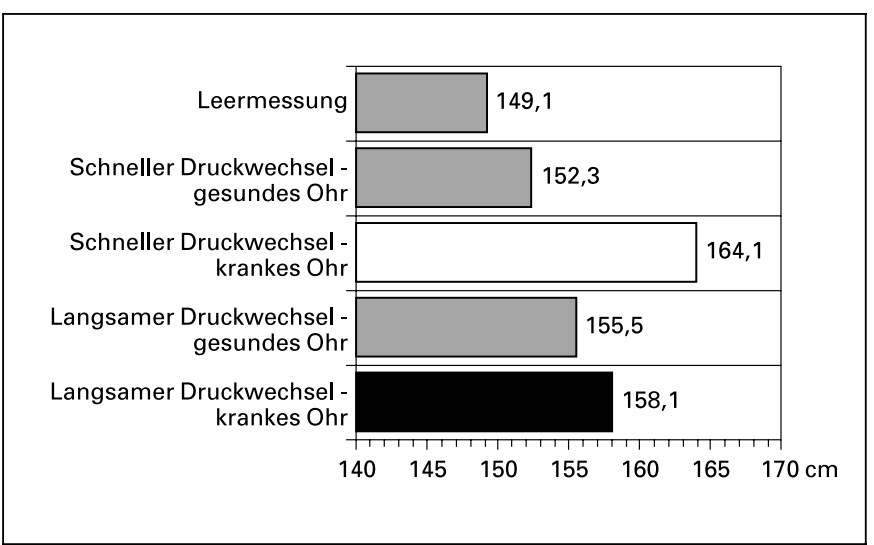

Abb. 3. Signifikante und hochsignifikante Zunahme der durchschnittlichen Schwankungslinie auf dem erkrankten Ohr im Vergleich zur Leermessung bei 19 Patienten mit M. Ménière.

Zur Untersuchung der vestibulookulären Reaktionen applizierten wir mit einem herkömmlichen Tympanometriegerät langsame und schnelle Druckwechsel zwischen -600 und $+400 \mathrm{~mm}$ daPa auf das gesunde und das erkrankte Ohr (Abb. 2, 3). Die langsamen Druckschwankungen wurden mit einer Druckänderungsgeschwindigkeit von $50 \mathrm{daPa} / \mathrm{s}$, die schnellen mit $600 \mathrm{da} / \mathrm{Pa} / \mathrm{s}$ verabreicht. Die Aufzeichnung der Augenbewegungen wurde mittels Elektronystagmographie mit einem 3-Kanal-ENG durchgeführt. Nach Eichung und Ableitung eines Ruhe-ENG wurden die horizontalen Augenbewegungen des rechten und linken Auges sowie die vertikalen Augenbewegungen des linken Auges unter langsamen und schnellen Druckschwankungen erfasst. Im Anschluss daran führten wir die Untersuchung der vestibulospinalen Reaktionen mit Hilfe der Posturographie (objektivierbarer Romberg-Test) auf einer sogenannten Vierfelderdruckmessplatte (Luzerner Messplatte) durch. Nach einer Probemessung zur Justierung auf den Probanden in der 0-Position des $x$-y-Feldes der Platte unter visueller Kontrolle und Abdunkelung des Raumes wurde eine Leermessung durchgeführt. Danach erfolgte in

Otorhinolaryngol Nova 2000;10:207-212 209 
Tabelle 1. Darstellung der signifikanten und hochsignifikanten Unterschiede im Wilcoxon-Test für 19 Patienten a Wilcoxon-Test (Matched-Pairs Signed Ranks Test)

\begin{tabular}{llll}
\hline & $\begin{array}{l}\text { Gesundes Ohr } \\
\text { langsam }\end{array}$ & $\begin{array}{l}\text { Krankes Ohr } \\
\text { schnell }\end{array}$ & $\begin{array}{l}\text { Gesundes Ohr } \\
\text { schnell }\end{array}$ \\
\hline $\begin{array}{l}\text { Krankes Ohr langsam } \\
\text { Gesundes Ohr langsam }\end{array}$ & 0,0413 & 0,0486 & \\
Krankes Ohr schnell & & & 0,4899 \\
\hline
\end{tabular}

b Wilcoxon-Test: Adjustierung des versuchsbezogenen $\alpha$ nach Bonferroni

\begin{tabular}{lllll}
\hline & $\begin{array}{l}\text { Krankes Ohr } \\
\text { langsam }\end{array}$ & $\begin{array}{l}\text { Gesundes Ohr } \\
\text { langsam }\end{array}$ & $\begin{array}{l}\text { Krankes Ohr } \\
\text { schnell }\end{array}$ & $\begin{array}{l}\text { Gesundes Ohr } \\
\text { schnell }\end{array}$ \\
\hline Leermessung & 0,0025 & 0,4380 & 0,0002 & 0,1841
\end{tabular}

Analogie zur ENG die Reizung beider Ohren mit langsamen und schnellen Druckschwankungen. Die Messdauer betrug jeweils $1 \mathrm{~min}$. Der Proband wurde aufgefordert, während dieser Zeit mit geschlossenen Augen so ruhig wie möglich zu stehen. Über einen PC wurde die Länge der posturographischen Schwankungslinie während der Messzeit für jede Messung einzeln registriert. Ausserdem wurden Abweichungen von der 0-Position und Zunahme der Schwankungsamplitude im Vergleich zur Leermessung beurteilt [9].

Zum statistischen Vergleich der Schwankungslinien wurde der (Paarvergleichs-) Test nach Wilcoxon herangezogen, die Adjustierung des versuchsbezogenen $\alpha$ erfolgte mit Hilfe des oben genannten Tests nach Bonferroni.

\section{Ergebnisse}

Das Durchschnittsalter der 19 untersuchten Patienten mit einseitigem M. Ménière betrug 51,1 Jahre (38-67 Jahre). Als durchschnittliche Erkrankungszeit wurden 10,8 Jahre (5-20 Jahre) ermittelt.

Neun Patienten (47,37\%) gaben auf Befragung an, gegenüber Luftdruckschwankungen, wie etwa Niesen, Naseputzen sowie beim Autofahren mit grösseren Höhenunterschieden, empfindlich, d.h. mit kurzzeitigem Unsicherheits- und Schwindelgefühl, zu reagieren (Gruppe A). Von den übrigen 10 Patienten $(52,63 \%)$ wurde Druckempfindlichkeit verneint (Gruppe B). Zwischen beiden Gruppen gab es im Hinblick auf das Durchschnittsalter sowie die durchschnittliche Erkrankungsdauer keine signifikanten Unterschiede.

Mittels der polygraphischen Elektronystagmographie waren weder bei langsamen noch bei schnellen Druckwechseln im ENG Nystagmen nachweisbar. Unterschiede zum Ruhe-ENG wurden nicht festgestellt. Zwischen erkrankter und gesunder Seite waren ebenfalls keine Unterschiede feststellbar.

Bei der Posturographie liessen sich allerdings Unterschiede der gemessenen Schwankungslinie nachweisen
(Tab. 1). Durchschnittlich betrug diese für die Leermessung $147 \mathrm{~cm}$. Bei langsamem und schnellem Druckwechsel wurden, jeweils für das kranke bzw. gesunde $\mathrm{Ohr}$ getrennt, 158,1 und $155,5 \mathrm{~cm}$ sowie 164,1 und $152,3 \mathrm{~cm}$ gemessen.

Im Wilcoxon-Test trat im Vergleich zur Leermessung ein signifikanter Unterschied zum kranken Ohr bei langsamem $(\alpha=0,0025)$ und ein hochsignifikanter Unterschied zum kranken Ohr bei schnellem Druckwechsel $(\alpha=$ 0,0002 ) auf. Die Unterschiede im gesunden Ohr waren dagegen sowohl bei langsamem $(\alpha=0,4380)$ als auch schnellem $(\alpha=0,1841)$ Druckwechsel nicht signifikant.

Im direkten Paarvergleich traten signifikante Differenzen zwischen krankem und gesundem Ohr bei langsamem Druckwechsel $(\alpha=0,0413)$, sowie zwischen langsamer und schneller Reizung des kranken Ohres $(\alpha=0,0486)$ auf. Der Unterschied bei schnellem Druckwechsel zwischen dem kranken und gesunden Ohr war sogar hochsignifikant $(\alpha=0,0003)$. Keine Signifikanz liess sich hingegen zwischen schneller und langsamer Reizung des gesunden Ohres ermitteln $(\alpha=0,4899)$.

Betrachtet man die x-y-Darstellung der Schwankungslinie im Hinblick auf Amplitudenerhöhungen und Abweichungen von der 0-Stellung im Vergleich zur Leermessung, lässt sich bei 13 der 19 Patienten $(68,42 \%)$ eine Verstärkung der Amplitude bei der schnellen Reizung des kranken Ohres verzeichnen. Bei 6 dieser Patienten war die Amplitude auch bei der langsamen Reizung des kranken Ohres erhöht. Fünf der 13 Patienten zeigten neben der Amplitudenerhöhung auch eine Abweichung von der 0 -Stellung. Allerdings war bei 2 Patienten auch eine Amplitudenverstärkung auch auf dem gesunden $\mathrm{Ohr}$ sowohl bei schnellem als auch bei langsamem Druckwechsel und bei 2 weiteren lediglich bei langsamer Reizung auf dem kranken Ohr sichtbar. 
Zwischen den Gruppen A und B zeigten sich keine deutlichen Unterschiede beim Vergleich der durchschnittlichen Schwankungslinien.

\section{Diskussion}

Einflüsse von unterschiedlichen Mittelohrdrucken auf Funktionen des Innenohrs, insbesondere Gleichgewichtsstörungen - auch als Hennebertsches Fistelsymptom bekannt - werden seit längerer Zeit unter verschiedenen Gesichtspunkten diskutiert.

Die Ursache wurde fast ausschliesslich in der direkten Druckübertragung über das runde Fenster gesucht. Durch Aufbau relativer statischer Überdrucke im Mittelohr liess sich eine Besserung des Hörvermögens einerseits bzw. eine Linderung der Vertigosymptomatik andererseits erzielen $[1,2]$. In anderen Untersuchungen liess sich bei Ménière-Patienten mit fluktuierender Tieftonschwerhörigkeit durch statischen Überdruck, der über eine Tympanometriesonde appliziert wurde, in Zeiten guten Hörens eine Depression der Knochenleitung bei $500 \mathrm{~Hz}$ erreichen. In Zeiten schlechten Hörens war hingegen keine Depression der Knochenleitungskurve zu beobachten [2]. Tierexperimentell konnte nachgewiesen werden, dass der im Innenohr aufbaubare Druck allerdings von nur sehr kurzer Dauer ist und nach wenigen Sekunden auf den Ausgangswert des Perilymphdrucks zurückgeht [10].

Wenig beachtet für die Druckübertragung wurde bisher die Bedeutung des im Vergleich zu den Innenohrfenstern sehr viel grösseren Trommelfells. Die grosse Fläche lässt durch den Hydraulikfaktor sehr viel grössere Kräfte entstehen, die über die Ossikelkette und das ovale Fenster in das Innenohr transportiert werden und erhebliche Perilymphschwankungen auslösen können. Im normalen Mittelohr werden diese Bewegungen und Kräfte von den gleitenden Ossikelgelenken abgefangen [4-6]. Bei einer behinderten Gleitfunktion der Ossikelkette, z.B. bei degenerativen Knorpelveränderungen, werden diese Kräfte jedoch fast unverändert in den hochsensiblen Drucksensor Innenohr übertragen. Denkbar wäre demnach, dass gerade die Rezeptoren der Vestibularorgane hierauf entweder akut oder unter der Dauerbelastung der fortwährend sich ändernden Umgebungsluftdrucke chronisch mit einer Änderung der Entladungsrate reagieren.

Ziel der durchgeführten Arbeit war es daher, durch Applikation positiver und negativer Drucke im Gehörgang Perilymphverschiebungen auszulösen und die vestibulookulären und vestibulospinalen Reaktionen zu untersuchen. Patienten mit einem länger bestehenden, einseitigen M. Ménière wurden ausgewählt, da bei diesen die Vestibularorgane möglicherweise sensibilisiert sind. Zudem wäre denkbar, dass bei einigen dieser Patienten die Symptomatik als Folge der chronischen Irritationen des

Der Einfluss statischen Mittelohrdrucks beim Morbus Ménière
Vestibularorgans durch die ungehindert einwirkenden Umgebungsluftdrucke entsteht.

In der ENG liessen sich sowohl auf dem gesunden als auf dem erkrankten Ohr weder mit schnellem noch mit langsamem Druckwechsel Nystagmusschläge provozieren. Daraus kann geschlossen werden, dass das vestibulookuläre System auf die Druckänderungen nur wenig sensibel reagiert. Andererseits kann man vermuten, dass mögliche Reaktionen mit dieser hierfür zu unempfindlichen Untersuchungsmethode nicht erfasst werden können.

Sensibler zeigte sich die Posturographie, was auch mit Erfahrungen aus der klinischen Praxis in Einklang steht. Es sind die vestibulospinalen Abweichreaktionen - das Gleichgewicht im engeren Sinn -, die den Patienten zuerst und in erster Linie störend auffallen [11]. So erwies sich die Posturographie im Intervall zwischen den Ménière-Anfällen als sensitivere Untersuchungsmethode im Vergleich zur ENG $[12,13]$. Es ist deshalb durchaus möglich, dass das Hennebertsche Fistelsymptom unter der Frenzelbrille negativ, auf der Luzerner Messplatte aber nachweisbar ist.

Die statistische Auswertung der Länge der Schwankungslinien zeigte trotz der geringen Unterschiede eine statistische Signifikanz. Bei langsamen und schnellen Druckänderungen kam es auf dem erkrankten Ohr zu einer signifikanten Längenzunahme der Schwankungslinie, wohingegen auf dem gesunden Ohr keine signifikante Verlängerung zu verzeichnen war. Ein schneller, d.h. dynamischer Druckwechsel stellt dabei verständlich den grösseren Reiz dar. Er führt zu einer signifikant grösseren Längenzunahme. Die subjektive Empfindung von Schwindelsensationen bei Druckänderungen im Alltag, etwa dem Naseputzen, die von rund der Hälfte der Patienten angegeben wurden, scheint aber auf die objektivierbaren vestibulospinalen Reaktionen keinen Einfluss zu nehmen. Der Vergleich der Schwankungslinien beider Gruppen ergab keine Unterschiede.

Wir schlussfolgern aus unseren Untersuchungen, dass das erkrankte Ohr bei Patienten mit $\mathrm{M}$. Ménière druckempfindlich ist, wobei das vestibulospinale Gleichgewichtssystem sensibler auf Druckschwankungen der Perilymphe reagiert als das vestibulookuläre. Die Posturographie kann somit gerade bei der Diagnostik unklarer Schwindelbeschwerden und zur Beurteilung der zentralen Kompensation eine wichtige Entscheidungshilfe darstellen und sollte einen festen Platz im Rahmen der Vestibularisdiagnostik einnehmen [14-16].

Es ist weiterhin zu diskutieren, ob eine gestörte Mikromechanik der Ossikelkette in Folge degenerativer Veränderungen bei der Pathogenese des M. Ménière mit eine Rolle spielt. Der beobachtete Umstand, dass schneller Druckwechsel schlechter als langsamer kompensiert wird, könnte ein Hinweis dafür sein. Einer langsamen Trom- 
melfellbewegung könnte der Gelenkmechanismus noch folgen, für schnelle wäre er dann allerdings zu steif. Es entstehen dann grössere Perilymphverschiebungen, die zu einer messbaren Reaktion des Vestibularorgans führen. Eine pathomechanische Beteiligung der Gehörknöchelchenkette würde auch die manchmal verblüffenden Erfolge einer Paukenröhrcheneinlage bei M. Ménière-Patienten erklären, da dadurch Trommelfellauslenkungen von vornherein vermieden werden $[17,18]$. Es können keine Trommelfellverlagerungen bei Änderungen des Umgebungsluftdruckes entstehen und druckinduzierte Innenohrsymptome bleiben aus. Ein vielleicht durch die Druckschwankungen chronisch gereiztes Vestibularorgan wird ruhiggestellt.
Ein Paukenröhrchen stellt jedoch keine Dauerlösung dar. Ein Ausstopfen der ovalen und runden Fensternische mit Bindegewebe und Fibrinkleber erhöht die «Steifheit» der Perilymphflüssigkeit, so dass bei diesen Patienten luftdruckausgelöste Verschiebungen der Perilymphe dauerhaft verringert werden können.

Diese Manipulationen bewirken auch bei manchen Patienten mit dem Verdacht auf eine Ruptur der runden Fenstermembran trotz unauffälliger anatomischer Verhältnisse im Bereich der Mittelohrfenster ein erstaunliches Sistieren der Schwindelsymptome. Vielleicht kann mit diesem kleinen Eingriff einigen M.-Ménière-Patienten bei mittelohrbedingter Pathogenese geholfen werden.

\section{References}

1 Densert O, Carlborg J, Stagg J: Transmission of low frequency pressure steps to the perilymphatic fluid. Acta Otolaryngol 1981;91:55-64.

2 Ingelstedt S, Ivarsson A, Tjernström Ö: Immediate relief of symptoms during acute attacks of Ménière's disease, using a pressure chamber. Acta Otolaryngol 1976;82:368-378.

3 Maier W, Beck C, Hauser R, Beck Chl: Die Auswirkungen einer Veränderung des Umgebungsdrucks auf den Spontannystagmus bei Patienten mit cochleovestibulären Erkrankungen. Laryngorhinootologie 1993;72:236241.

4 Hüttenbrink K-B: Die Mechanik der Gehörknöchelchen bei statischen Drucken. II. Behinderte Gelenkfuntion und operative Kettenkonstruktion. Laryngol Rhinol Otol 1988;67:100105.

5 Hüttenbrink K-B: Die Mechanik und Funktion des Mittelohres. Teil 1: Die Ossikelkette und die Mittelohrmuskeln. Laryngorhinootologie 1992;71:545-551.
6 Hüttenbrink K-B: Die Funktion der Gehörknöchelchen und der Muskeln des Mittelohres. Eur Arch Otorhinolaryngol Suppl 1995;1:1-52.

7 Belal A, Stewart TJ: Pathological changes in the middle ear joints. Ann Otol Rhinol Laryngol 1960;83:159-167.

8 Etholm B, Belal A Jr: Senile changes in the middle ear joints. Ann Otol Rhinol Larnygol 1974;83:49-54.2.

9 Fried R, Arnold W: Der objektivierbare Rombergtest (Posturographie) mit der neuen «Luzerner Messplatte». Laryngorhinol Otol 1987; 66:433-436.

10 Carlborg B, Farmer JC, Carlborg A: Effects of hypobaric pressure on the labyrinth. Acta Otolaryngol 1990;110:386-393.

11 Densert O, Ingelstedt S, Ivarsson A, Pedersen $\mathrm{K}$ : Immediate restoration of basal sensorineural hearing (Mb.Ménière) using a pressure chamber. Acta Otolaryngol 1975;80:93-100.

12 Keim RJ: Clinical comparisons of posturography and electronystagmography. Laryngoscope 1993; 103:713-716.
13 Hytonen M, Pyykko I, Aalto H, Juhola M, Ramsay H: Vestibulo-ocular and vestibulo-spinal reflexes in evaluation of vestibular lesions. Acta Otolaryngol Suppl 1989;468:231-234.

14 Di Fabio RP: Sensitivity and specificity of platform posturography for identifying patients with vestibular dysfunction. Phys Ther 1995; 75:290-305.

15 Voorhees RL: The role of the dynamic posturography in neurotologic diagnosis. Laryngoscope 1989;99:995-1001.

16 Black FO, Wade SW, Nashner LM: What is the minimal vestibular function required for compensation? Am J Otol 1996;17:401-409.

17 Häusler R, Guillemin P. Traitement chirurgical de la maladie de Ménière par sacculotomie et aérateurs transtympaniques. Rev Laryngol 1991;112:2.

18 Montandon P, Guillemin P, Häusler R: Treatment of Ménière's disease by minor surgical procedures: Sacculotomy, cochleosacculotomy, transtympanic ventilation tubes. ORL 1988; $13: 416-422$. 